ROCKY MOUNTAIN

JOURNAL OF MATHEMATICS

Volume 45, Number 4, 2015

\title{
GRÖBNER-SHIRSHOV BASES OF SOME WEYL GROUPS
}

\author{
EYLEM GÜZEL KARPUZ, FIRAT ATEŞ AND A. SINAN ÇEVIK
}

\begin{abstract}
In this paper, we obtain Gröbner-Shirshov (non-commutative) bases for the $n$-extended affine Weyl group $\widetilde{W}$ of type $A_{1}$, elliptic Weyl groups of types $A_{1}^{(1,1)}$, $A_{1}^{(1,1)^{*}}$ and the 2-extended affine Weyl group of type $A_{2}^{(1,1)}$ with a generator system of a 2-toroidal sense. It gives a new algorithm for getting normal forms of elements of these groups and hence a new algorithm for solving the word problem in these groups.
\end{abstract}

1. Introduction. The Gröbner basis theory for commutative algebras was introduced by Buchberger [8] and provides a solution to the reduction problem for commutative algebras. In [3], Bergman generalized the Gröbner basis theory to associative algebras by proving the "Diamond lemma." On the other hand, the parallel theory of Gröbner bases was developed for Lie algebras by Shirshov [14]. In [4], Bokut noticed that Shirshov's method also works for associative algebras. Hence, for this reason, Shirshov's theory for Lie algebras and their universal enveloping algebras is called the Gröbner-Shirshov basis theory. There are some important studies on this subject related to the groups (see, for instance, $[\mathbf{7}, \mathbf{9}])$. We may finally refer to the papers $[\mathbf{2}, \mathbf{5}, \mathbf{6}, \mathbf{1 0}, \mathbf{1 1}]$ for some other recent studies on Gröbner-Shirshov bases.

Algorithmic problems such as the word, conjugacy and isomorphism problems have played an important role in group theory since the work of Dehn in the early 1900's. These problems are called decision problems which ask for a yes or no answer to a specific question. Among these decision problems, the word problem especially has been studied widely in groups (see [1]). It is well known that the word problem for finitely presented groups is not solvable in general; that is, given

2010 AMS Mathematics subject classification. Primary 13P10, Secondary $20 \mathrm{~F} 55$.

Keywords and phrases. Gröbner-Shirshov basis, presentation, Weyl group.

Received by the editors on September 22, 2012, and in revised form on March 8, 2013.

DOI: $10.1216 /$ RMJ-2015-45-4-1165

Copyright (C)2015 Rocky Mountain Mathematics Consortium 
any two words obtained by generators of the group, there may be no algorithm to decide whether these words represent the same element in this group.

The method of Gröbner-Shirshov bases which is the main theme of this paper gives a new algorithm for getting normal forms of elements of groups, and hence a new algorithm for solving the word problem in these groups. By considering this fact, our aim in this paper is to find Gröbner-Shirshov bases of the $n$-extended affine Weyl group of type $A_{1}$, elliptic Weyl groups of types $A_{1}^{(1,1)}, A_{1}^{(1,1)^{*}}$ and the 2-extended affine Weyl group of type $A_{2}^{(1,1)}$.

Extended affine root systems and the associated Weyl groups were introduced and studied by Saito [12]. In particular, 2-extended affine root systems are also called elliptic root systems from the point of view of the elliptic singularities. The defining relations of generators of the elliptic Weyl groups associated to the elliptic root systems were obtained by Saito and Takebayashi [13].

Throughout this paper, we order words in given alphabet in the deg-lex way comparing two words first by their lengths and then lexicographically when the lengths are equal. Additionally, $(i) \cap(j)$ and $(i) \cup(j)$ denote the intersection and inclusion compositions of relations $(i),(j)$, respectively.

2. Gröbner-Shirshov bases and composition-diamond lemma. Let $K$ be a field and $K\langle X\rangle$ the free associative algebra over $K$ generated by $X$. Denote $X^{*}$ as the free monoid generated by $X$, where the empty word is the identity denoted by 1 . For a word $w \in X^{*}$, we denote the length of $w$ by $|w|$. Suppose that $X^{*}$ is a well-ordered set. Then every nonzero polynomial $f \in K\langle X\rangle$ has the leading word $\bar{f}$. If the coefficient of $\bar{f}$ in $f$ is equal to 1 , then $f$ is called monic.

Let $f$ and $g$ be two monic polynomials in $K\langle X\rangle$. We then have two compositions as follows:

- If $w$ is a word such that $w=\bar{f} b=a \bar{g}$ for some $a, b \in X^{*}$ with $|\bar{f}|+|\bar{g}|>|w|$, then the polynomial $(f, g)_{w}=f b-a g$ is called the intersection composition of $f$ and $g$ with respect to $w$. The word $w$ is called an ambiguity of intersection.

- If $w=\bar{f}=a \bar{g} b$ for some $a, b \in X^{*}$, then the polynomial 
$(f, g)_{w}=f-a g b$ is called the inclusion composition of $f$ and $g$ with respect to $w$. The word $w$ is called an ambiguity of inclusion.

If $g$ is monic, $\bar{f}=a \bar{g} b$ and $\alpha$ is the coefficient of the leading term $\bar{f}$, then the transformation $f \mapsto f-\alpha a g b$ is called elimination (ELW) of the leading word of $g$ in $f$.

Let $S \subseteq K\langle X\rangle$ with each $s \in S$ monic. Then the composition $(f, g)_{w}$ is called trivial modulo $(S, w)$ if $(f, g)_{w}=\sum \alpha_{i} a_{i} s_{i} b_{i}$, where each $\alpha_{i} \in K, a_{i}, b_{i} \in X^{*}, s_{i} \in S$ and $a_{i} \overline{s_{i}} b_{i}<w$. If this is the case, then we write $(f, g)_{w} \equiv 0 \bmod (S, w)$.

We call the set $S$ endowed with the well ordering < a GröbnerShirshov basis for $K\langle X \mid S\rangle$ if any composition $(f, g)_{w}$ of polynomials in $S$ is trivial modulo $S$ and corresponding $w$.

The following lemma was proved by Shirshov [14] for free Lie algebras with deg-lex ordering.

Lemma 2.1. (Composition-Diamond lemma). Let $K$ be a field, let $A=K\langle X \mid S\rangle=K\langle X\rangle / \operatorname{Id}(S)$, and let $<$ be a monomial ordering on $X^{*}$, where $\operatorname{Id}(S)$ is the ideal of $K\langle X\rangle$ generated by $S$. Then the following statements are equivalent:

(i) $S$ is a Gröbner-Shirshov basis.

(ii) $f \in \operatorname{Id}(S) \Rightarrow \bar{f}=a \bar{s} b$ for some $s \in S$ and $a, b \in X^{*}$.

(iii) $\operatorname{Irr}(S)=\left\{u \in X^{*} \mid u \neq a \bar{s} b, s \in S, a, b \in X^{*}\right\}$ is a basis for the algebra $A=K\langle X \mid S\rangle$.

If a subset $S$ of $K\langle X\rangle$ is not a Gröbner-Shirshov basis, then we can add to $S$ all nontrivial compositions of polynomials of $S$, and by continuing this process (maybe infinitely) many times, we eventually obtain a Gröbner-Shirshov basis $S^{\text {comp }}$. Such a process is called the Shirshov algorithm.

2.1. Gröbner-Shirshov basis for the $n$-extended affine Weyl group $\widetilde{W}$ of type $A_{1}$. In [17], the author calculated the growth series of the $n$-extended affine Weyl group $\widetilde{W}$ of type $A_{1}$ with a generator system of an $n$-toroidal sense. To do that the author had the following result. 
Proposition 2.2. [17]. The n-extended affine Weyl group $\widetilde{W}$ of type $A_{1}$ is presented as follows:

Generators: $w_{i}(0 \leq i \leq n)$,

Relations: $w_{i}^{2}=1(0 \leq i \leq n),\left(w_{i} w_{1} w_{j}\right)^{2}=1(i, j \neq 1,0 \leq i \neq j \leq$ $n)$.

Let us order the generators as in the following:

- for $i<j$, we have $w_{i}>w_{j}$, i.e., $w_{0}>w_{1}>w_{2}>\cdots>w_{n}$.

Now we give the first main result of this section.

Theorem 2.3. A Gröbner-Shirshov basis of the n-extended affine Weyl group $\widetilde{W}$ of type $A_{1}$ consists of the following polynomials:

$$
\begin{aligned}
& \text { (1) } w_{i}^{2}-1 \quad(0 \leq i \leq n), \\
& \text { (2) } w_{i} w_{1} w_{j}-w_{j} w_{1} w_{i} \quad(i, j \neq 1,0 \leq i \neq j \leq n),
\end{aligned}
$$

relative to the deg-lex order of words in the generators.

Proof. We need to prove that all compositions of polynomials (1)-(2) are trivial. To do that, firstly, we consider the intersection compositions of these polynomials. Hence, we have the following ambiguities $w$ :

$$
\begin{aligned}
& (1) \cap(1): w=w_{i}^{3} \quad(0 \leq i \leq n), \\
& (1) \cap(2): w=w_{i}^{2} w_{1} w_{j} \quad(i, j \neq 1,0 \leq i \neq j \leq n), \\
& (2) \cap(1): w=w_{i} w_{1} w_{j}^{2} \quad(i, j \neq 1,0 \leq i \neq j \leq n), \\
& (2) \cap(2): w=w_{i} w_{1} w_{j} w_{1} w_{k} \quad(i, j, k \neq 1,0 \leq i<j<k \leq n) .
\end{aligned}
$$

All these ambiguities are trivial. Let us show one of them.

$$
\begin{aligned}
(2) \cap(2): w= & w_{i} w_{1} w_{j} w_{1} w_{k} \quad(i, j, k \neq 1,0 \leq i<j<k \leq n), \\
(f, g)_{w}= & \left(w_{i} w_{1} w_{j}-w_{j} w_{1} w_{i}\right) w_{1} w_{k}-w_{i} w_{1}\left(w_{j} w_{1} w_{k}-w_{k} w_{1} w_{j}\right) \\
= & w_{i} w_{1} w_{j} w_{1} w_{k}-w_{j} w_{1} w_{i} w_{1} w_{k}-w_{i} w_{1} w_{j} w_{1} w_{k} \\
& +w_{i} w_{1} w_{k} w_{1} w_{j} \\
= & w_{i} w_{1} w_{k} w_{1} w_{j}-w_{j} w_{1} w_{i} w_{1} w_{k} \\
& \quad\left(\text { for } i<k \text { we have } w_{i} w_{1} w_{k}-w_{k} w_{1} w_{i}\right) \\
\equiv & w_{k} w_{1} w_{i} w_{1} w_{j}-w_{j} w_{1} w_{k} w_{1} w_{i} \\
& \quad\left(\text { for } j<k \text { we have } w_{j} w_{1} w_{k}-w_{k} w_{1} w_{j}\right)
\end{aligned}
$$




$$
\equiv w_{k} w_{1} w_{j} w_{1} w_{i}-w_{k} w_{1} w_{j} w_{1} w_{i} \equiv 0
$$

It is seen that there are no inclusion compositions of polynomials (1)-(2). Hence, the proof.

By considering Lemma 2.1 and Theorem 2.3, we have the following result.

Corollary 2.4. Let $C(u)$ be a normal form of a word $u \in A_{1}$. Then $C(u)$ has a form

$$
\begin{gathered}
w_{i_{1}}^{\epsilon_{i_{1}}} w_{i_{2}}^{\epsilon_{i_{2}}} \cdots w_{i_{k}}^{\epsilon_{i_{k}}} w_{1} w_{j_{1}}^{\epsilon_{j_{1}}} w_{j_{2}}^{\epsilon_{j_{2}}} \cdots w_{j_{t}}^{\epsilon_{j_{t}}} w_{1} w_{s_{1}}^{\epsilon_{s_{1}}} w_{s_{2}}^{\epsilon_{s_{2}}} \cdots w_{s_{r}}^{\epsilon_{s_{r}}} w_{1} \\
\cdots w_{1} w_{m_{1}}^{\epsilon_{m_{1}}} w_{m_{2}}^{\epsilon_{m_{2}}} \cdots w_{m_{p}}^{\epsilon_{m_{p}}} w_{1} w_{n_{1}}^{\epsilon_{n_{1}}} w_{n_{2}}^{\epsilon_{n_{2}}} \cdots w_{n_{l}}^{\epsilon_{n_{l}}}
\end{gathered}
$$

where $i_{k}<j_{1}, j_{t}<s_{1}, m_{p}<n_{1}$ and $\epsilon_{q}=0,1$ for $q \in\left\{i_{1}, \ldots, i_{k}, j_{1}, \ldots, j_{t}\right.$, $\left.s_{1}, \ldots, s_{r}, m_{1}, \ldots, m_{p}, n_{1}, \ldots, n_{l}\right\}$.

\subsection{Gröbner-Shirshov basis for the elliptic Weyl groups of} types $A_{1}^{(1,1)}$ and $A_{1}^{(1,1) *}$. The generators and relations of the elliptic Weyl group $W$ of type $A_{1}^{(1,1)}$ are given as follows ([13], [16]):

Generators: $w_{i}, w_{i}^{*}(i=0,1)$,

Relations: $w_{i}^{2}=w_{i}^{* 2}=1(i=0,1), w_{0} w_{0}^{*} w_{1} w_{1}^{*}=1$.

The relation $w_{0} w_{0}^{*} w_{1} w_{1}^{*}=1$ is also written as $w_{0}^{*} w_{1}=w_{0} w_{1}^{*}(\Leftrightarrow$ $\left.w_{1}^{*} w_{0}=w_{1} w_{0}^{*}\right)$.

Now we order the generators as $w_{0}^{*}>w_{0}>w_{1}^{*}>w_{1}$. Therefore, we have the following result.

Theorem 2.5. A Gröbner-Shirshov basis of the elliptic Weyl group $W$ of type $A_{1}^{(1,1)}$ consists of the following polynomials:
(1) $w_{0}^{2}-1$,
(2) $w_{1}^{2}-1$,
(3) $w_{0}^{* 2}-1$,
(4) $w_{1}^{* 2}-1$,
(5) $w_{0}^{*} w_{1}-w_{0} w_{1}^{*}$,
(6) $w_{0}^{*} w_{0}-w_{1} w_{1}^{*}$,

relative to deg-lex order of words in the generators.

Proof. We need to prove that all compositions of polynomials (1)-(6) are trivial. To do that, firstly, we consider the intersection compositions 
of these polynomials. Thus we have the following ambiguities:
(1) $\cap(1): w=w_{0}^{3}$,
$(2) \cap(2): w=w_{1}^{3}$,
(3) $\cap(3): w=w_{0}^{* 3}$,
(4) $\cap(4): w=w_{1}^{* 3}$,
$(3) \cap(5): w=w_{0}^{* 2} w_{1}$,
$(5) \cap(2): w=w_{0}^{*} w_{1}^{2}$,
(3) $\cap(6): w=w_{0}^{* 2} w_{0}$,
$(6) \cap(1): w=w_{0}^{*} w_{0}^{2}$.

All of these ambiguities are trivial. Let us show some of them.

$$
\begin{aligned}
(1) \cap(1): w & =w_{0}^{3}, \\
(f, g)_{w} & =\left(w_{0}^{2}-1\right) w_{0}-w_{0}\left(w_{0}^{2}-1\right) \\
& =w_{0}^{3}-w_{0}-w_{0}^{3}+w_{0} \equiv 0 . \\
(3) \cap(5): w & =w_{0}^{* 2} w_{1}, \\
(f, g)_{w} & =\left(w_{0}^{* 2}-1\right) w_{1}-w_{0}^{*}\left(w_{0}^{*} w_{1}-w_{0} w_{1}^{*}\right) \\
& =w_{0}^{* 2} w_{1}-w_{1}-w_{0}^{* 2} w_{1}+w_{0}^{*} w_{0} w_{1}^{*} \\
& =w_{0}^{*} w_{0} w_{1}^{*}-w_{1} \equiv w_{1} w_{1}^{* 2}-w_{1} \equiv 0 .
\end{aligned}
$$

It is seen that there are no inclusion compositions of polynomials (1)-(6). Hence, the proof.

By Lemma 2.1 and Theorem 2.5, we have the following result.

Corollary 2.6. Let $C(u)$ be a normal form of a word $u \in A_{1}^{(1,1)}$. Then $C(u)$ has a form $U_{1}\left(w_{0}^{*}\right)^{n_{1}}\left(w_{1}^{*}\right)^{n_{1}{ }^{\prime}} U_{2}\left(w_{0}^{*}\right)^{n_{2}}\left(w_{1}^{*}\right)^{n_{2}{ }^{\prime}} \cdots U_{k}\left(w_{0}^{*}\right)^{n_{k}}\left(w_{1}^{*}\right)^{n_{k}{ }^{\prime}}$, where $n_{i}, n_{i}{ }^{\prime}=0,1$ and $U_{i}=w_{0}^{\epsilon_{i_{1}}} w_{1}^{\delta_{i_{1}}} w_{0}^{\epsilon_{i_{2}}} w_{1}^{\delta_{i_{2}}} \cdots w_{0}^{\epsilon_{i_{s}}} w_{1}^{\delta_{i_{s}}}$, where $\epsilon_{i_{j}}, \delta_{i_{j}}=0,1$ for $1 \leq i \leq k$ and $1 \leq j \leq s$.

Another elliptic Weyl group $A_{1}^{(1,1) *}$ has the following generators and relations:

Generators: $w_{0}, w_{1} w_{1}^{*}$,

Relations: $w_{0}^{2}=w_{1}^{2}=w_{1}^{* 2}=\left(w_{0} w_{1} w_{1}^{*}\right)^{2}=1$.

This Weyl group is obtained from the Weyl group of type $A_{1}^{(1,1)}$ by removing one generator $w_{0}^{*}$.

Now we order the generators as $w_{0}>w_{1}^{*}>w_{1}$. According to this order, we have the following result, the proof of which can be done easily and similarly to the proof of Theorem 2.5. 
Theorem 2.7. A Gröbner-Shirshov basis of the elliptic Weyl group $W$ of type $A_{1}^{(1,1) *}$ consists of the following polynomials:
(1) $w_{0}^{2}-1$,
(2) $w_{1}^{2}-1$
(3) $w_{1}^{* 2}-1$,
(4) $w_{0} w_{1} w_{1}^{*}-w_{1}^{*} w_{1} w_{0}$

relative to deg-lex order of words in the generators.

By Lemma 2.1 and Theorem 2.7, we have the following result.

Corollary 2.8. Let $C(u)$ be a normal form of a word $u \in A_{1}^{(1,1) *}$. Then $C(u)$ has a form $U_{1}\left(w_{1}^{*}\right)^{n_{1}} U_{2}\left(w_{1}^{*}\right)^{n_{2}} \cdots U_{t}\left(w_{1}^{*}\right)^{n_{t}}$, where $n_{i}=0,1$ and $U_{i}=w_{0}^{\epsilon_{i_{1}}} w_{1}^{\delta_{i_{1}}} w_{0}^{\epsilon_{i_{2}}} w_{1}^{\delta_{i_{2}}} \cdots w_{0}^{\epsilon_{i_{p}}} w_{1}^{\delta_{i_{p}}}$, where $\epsilon_{i_{j}}, \delta_{i_{j}}=0,1$, for $1 \leq i \leq t$ and $1 \leq j \leq p$.

2.3. Gröbner-Shirshov basis for the 2-extended affine Weyl group $\widetilde{W}$ of type $A_{2}^{(1,1)}$. The Weyl groups, the elliptic Weyl group of type $A_{2}^{(1,1)}$ in [18] and the 2-extended affine Weyl group of type $A_{2}^{(1,1)}$ in this paper are isomorphic, but their generator systems are different, and the latter is obtained by removing two generators from the former.

Proposition 2.9. [15]. The 2-extended affine Weyl group $\widetilde{W}$ of type $A_{2}^{(1,1)}$ is presented as follows:

Generators: $w_{i}(0 \leq i \leq 3)$,

Relations: $w_{i}^{2}=1(0 \leq i \leq 3)$,

$$
\begin{array}{ll}
w_{0} w_{1} w_{0}=w_{1} w_{0} w_{1}, & w_{0} w_{2} w_{0}=w_{2} w_{0} w_{2}, \\
w_{1} w_{2} w_{1}=w_{2} w_{1} w_{2}, & w_{1} w_{3} w_{1}=w_{3} w_{1} w_{3}, \\
w_{2} w_{3} w_{2}=w_{3} w_{2} w_{3}, & w_{0} w_{1} w_{0} w_{2} w_{3}=w_{3} w_{1} w_{0} w_{2} w_{0}
\end{array}
$$

Let us order the generators as $w_{0}>w_{1}>w_{2}>w_{3}$. Now we have the following result. 
Theorem 2.10. A Gröbner-Shirshov basis of the 2-extended affine Weyl group $\widetilde{W}$ of type $A_{2}^{(1,1)}$ consists of the following polynomials:
(1) $w_{0}^{2}-1$,
(2) $w_{1}^{2}-1$,
(3) $w_{2}^{2}-1$,
(4) $w_{3}^{2}-1$,
(5) $w_{0} w_{1} w_{0}-w_{1} w_{0} w_{1}$,
(6) $w_{0} w_{2} w_{0}-w_{2} w_{0} w_{2}$
(7) $w_{1} w_{2} w_{1}-w_{2} w_{1} w_{2}$
(8) $w_{1} w_{3} w_{1}-w_{3} w_{1} w_{3}$
(9) $w_{2} w_{3} w_{2}-w_{3} w_{2} w_{3}$,
(10) $w_{0} w_{1} w_{0} w_{2} w_{3}-w_{3} w_{1} w_{0} w_{2} w_{0}$

(11) $w_{0} w_{3} w_{1} w_{3} w_{2}-w_{1} w_{3} w_{2} w_{3} w_{0}$,

(12) $w_{1} w_{0} w_{1} w_{3} w_{2}-w_{3} w_{1} w_{2} w_{0} w_{3}$

relative to deg-lex order of words in the generators.

Proof. We need to prove that all compositions of polynomials (1)(12) are trivial. To do that, firstly, we consider the intersection compositions of these polynomials. Thus we have the following ambiguities:
$(1) \cap(1): w=w_{0}^{3}$,
$(1) \cap(5): w=w_{0}^{2} w_{1} w_{0}$,

$$
(1) \cap(6): w=w_{0}^{2} w_{2} w_{0},
$$

(1) $\cap(10): w=w_{0}^{2} w_{1} w_{0} w_{2} w_{3}$,

(1) $\cap(11): w=w_{0}^{2} w_{3} w_{1} w_{3} w_{2}$,

$(2) \cap(2): w=w_{1}^{3}$,

$(2) \cap(7): w=w_{1}^{2} w_{2} w_{1}$,

$(2) \cap(8): w=w_{1}^{2} w_{3} w_{1}$,

$(2) \cap(12): w=w_{1}^{2} w_{0} w_{1} w_{3} w_{2}$,

$(3) \cap(3): w=w_{2}^{3}$,

$(3) \cap(9): w=w_{2}^{2} w_{3} w_{2}$,

$(4) \cap(4): w=w_{3}^{3}$,

$(5) \cap(1): w=w_{0} w_{1} w_{0}^{2}$,

$(5) \cap(5): w=w_{0} w_{1} w_{0} w_{1} w_{0}$,

$(5) \cap(6): w=w_{0} w_{1} w_{0} w_{2} w_{0}$,

$(5) \cap(10): w=w_{0} w_{1} w_{0} w_{1} w_{0} w_{2} w_{3}$,

$(5) \cap(11): w=w_{0} w_{1} w_{0} w_{3} w_{1} w_{3} w_{2}$,

$(6) \cap(1): w=w_{0} w_{2} w_{0}^{2}$,

$(6) \cap(5): w=w_{0} w_{2} w_{0} w_{1} w_{0}$,

$(6) \cap(6): w=w_{0} w_{2} w_{0} w_{2} w_{0}$,

$(6) \cap(10): w=w_{0} w_{2} w_{0} w_{1} w_{0} w_{2} w_{3}$,

$(6) \cap(11): w=w_{0} w_{2} w_{0} w_{3} w_{1} w_{3} w_{2}$,

$(7) \cap(2): w=w_{1} w_{2} w_{1}^{2}$,

$(7) \cap(7): w=w_{1} w_{2} w_{1} w_{2} w_{1}$,

$(7) \cap(8): w=w_{1} w_{2} w_{1} w_{3} w_{1}$,

$(7) \cap(12): w=w_{1} w_{2} w_{1} w_{0} w_{1} w_{3} w_{2}$,

$(8) \cap(2): w=w_{1} w_{3} w_{1}^{2}$,

$(8) \cap(7): w=w_{1} w_{3} w_{1} w_{2} w_{1}$,

$(8) \cap(8): w=w_{1} w_{3} w_{1} w_{3} w_{1}$,

$(8) \cap(12): w=w_{1} w_{3} w_{1} w_{0} w_{1} w_{3} w_{2}$,

$(9) \cap(3): w=w_{2} w_{3} w_{2}^{2}$, 
$(9) \cap(9): w=w_{2} w_{3} w_{2} w_{3} w_{2}$,

$(10) \cap(4): w=w_{0} w_{1} w_{0} w_{2} w_{3}^{2}$,

$(11) \cap(3): w=w_{0} w_{3} w_{1} w_{3} w_{2}^{2}$,

$(11) \cap(9): w=w_{0} w_{3} w_{1} w_{3} w_{2} w_{3} w_{2}$,

$(12) \cap(3): w=w_{1} w_{0} w_{1} w_{3} w_{2}^{2}$,

$(12) \cap(9): w=w_{1} w_{0} w_{1} w_{3} w_{2} w_{3} w_{2}$.

All of these ambiguities are trivial. Let us show some of them.

$(2) \cap(7): w=w_{1}^{2} w_{2} w_{1}$,

$$
\begin{aligned}
(f, g)_{w} & =\left(w_{1}^{2}-1\right) w_{2} w_{1}-w_{1}\left(w_{1} w_{2} w_{1}-w_{2} w_{1} w_{2}\right) \\
& =w_{1}^{2} w_{2} w_{1}-w_{2} w_{1}-w_{1}^{2} w_{2} w_{1}+w_{1} w_{2} w_{1} w_{2} \\
& =w_{1} w_{2} w_{1} w_{2}-w_{2} w_{1} \equiv w_{2} w_{1} w_{2}^{2}-w_{2} w_{1} \equiv 0 .
\end{aligned}
$$

$(12) \cap(9): w=w_{1} w_{0} w_{1} w_{3} w_{2} w_{3} w_{2}$,

$$
\begin{aligned}
(f, g)_{w}= & \left(w_{1} w_{0} w_{1} w_{3} w_{2}-w_{3} w_{1} w_{2} w_{0} w_{3}\right) w_{3} w_{2} \\
& -w_{1} w_{0} w_{1} w_{3}\left(w_{2} w_{3} w_{2}-w_{3} w_{2} w_{3}\right) \\
= & w_{1} w_{0} w_{1} w_{3} w_{2} w_{3} w_{2}-w_{3} w_{1} w_{2} w_{0} w_{3}^{2} w_{2} \\
& -w_{1} w_{0} w_{1} w_{3} w_{2} w_{3} w_{2}+w_{1} w_{0} w_{1} w_{3}^{2} w_{2} w_{3} \\
\equiv & w_{1} w_{0} w_{1} w_{2} w_{3}-w_{3} w_{1} w_{2} w_{0} w_{2} \equiv w_{3} w_{1} w_{2} w_{0} w_{2} \\
& -w_{3} w_{1} w_{2} w_{0} w_{2} \equiv 0 .
\end{aligned}
$$

Now we consider the ambiguity $(5) \cap(6): w=w_{0} w_{1} w_{0} w_{2} w_{0}$. Then we get

$$
\begin{aligned}
(f, g)_{w} & =\left(w_{0} w_{1} w_{0}-w_{1} w_{0} w_{1}\right) w_{2} w_{0}-w_{0} w_{1}\left(w_{0} w_{2} w_{0}-w_{2} w_{0} w_{2}\right) \\
& =w_{0} w_{1} w_{0} w_{2} w_{0}-w_{1} w_{0} w_{1} w_{2} w_{0}-w_{0} w_{1} w_{0} w_{2} w_{0}+w_{0} w_{1} w_{2} w_{0} w_{2} \\
& =w_{0} w_{1} w_{2} w_{0} w_{2}-w_{1} w_{0} w_{1} w_{2} w_{0}
\end{aligned}
$$

The polynomial $w_{0} w_{1} w_{2} w_{0} w_{2}-w_{1} w_{0} w_{1} w_{2} w_{0}$ is written as the relator $w_{0} w_{1} w_{2} w_{0} w_{2}=w_{1} w_{0} w_{1} w_{2} w_{0}$. Since we have studied group structure and we have the relator $w_{0}^{2}=1$, we can multiply both sides of this relation by $w_{0}$. Hence, we obtain the relation $w_{0} w_{1} w_{2} w_{0} w_{2} w_{0}=$ $w_{1} w_{0} w_{1} w_{2}$, and thus the polynomial $w_{0} w_{1} w_{2} w_{0} w_{2} w_{0}-w_{1} w_{0} w_{1} w_{2}$. Then we get

$$
\begin{aligned}
w_{0} w_{1} w_{2} w_{0} w_{2} w_{0}-w_{1} w_{0} w_{1} w_{2} & \equiv w_{0} w_{1} w_{2}^{2} w_{0} w_{2}-w_{1} w_{0} w_{1} w_{2} \\
& \equiv w_{0} w_{1} w_{0} w_{2}-w_{1} w_{0} w_{1} w_{2} \\
& \equiv w_{1} w_{0} w_{1} w_{2}-w_{1} w_{0} w_{1} w_{2} \equiv 0
\end{aligned}
$$


The ambiguities $(5) \cap(11),(6) \cap(5),(6) \cap(10),(6) \cap(11),(7) \cap(8),(7) \cap$ (12), (8) $\cap(7),(8) \cap(12),(11) \cap(3),(11) \cap(9),(12) \cap(3)$ are also trivial by the same process.

Now we check inclusion compositions of polynomials (1)-(12). In this case, we have one inclusion composition (5) $\cup(10): w=$ $w_{0} w_{1} w_{0} w_{2} w_{3}$ which is trivial. Let us show it.

$$
\begin{aligned}
(5) \cup(10): w= & w_{0} w_{1} w_{0} w_{2} w_{3}, \\
(f, g)_{w}= & \left(w_{0} w_{1} w_{0}-w_{1} w_{0} w_{1}\right) w_{2} w_{3} \\
& -1\left(w_{0} w_{1} w_{0} w_{2} w_{3}-w_{3} w_{1} w_{0} w_{2} w_{0}\right) \\
= & w_{0} w_{1} w_{0} w_{2} w_{3}-w_{1} w_{0} w_{1} w_{2} w_{3} \\
& -w_{0} w_{1} w_{0} w_{2} w_{3}+w_{3} w_{1} w_{0} w_{2} w_{0} \\
= & w_{3} w_{1} w_{0} w_{2} w_{0}-w_{1} w_{0} w_{1} w_{2} w_{3} \\
\equiv & w_{3} w_{1} w_{0} w_{2} w_{0}-w_{3} w_{1} w_{0} w_{2} w_{0} \equiv 0 .
\end{aligned}
$$

Hence, the proof.

By considering Theorems 2.3, 2.5, 2.7 and 2.10, we have the following result.

Corollary 2.11. The word problem for the n-extended affine Weyl group of type $A_{1}$, elliptic Weyl groups of types $A_{1}^{(1,1)}, A_{1}^{(1,1)^{*}}$ and the 2-extended affine Weyl group of type $A_{2}^{(1,1)}$ is solvable.

Acknowledgments. The authors would like to thank the referee for his/her kind suggestions and valuable comments that improved the understandable of this paper.

\section{REFERENCES}

1. S.I. Adian and V.G. Durnev, Decision problems for groups and semigroups, Russian Math. Surv. 55 (2000), 207-296.

2. F. Ateş, E.G. Karpuz, C. Kocapinar and A.S. Çevik, Gröbner-Shirshov bases of some monoids, Discr. Math. 311 (2011), 1064-1071.

3. G.M. Bergman, The diamond lemma for ring theory, Adv. Math. 29 (1978), $178-218$.

4. L.A. Bokut, Imbedding into simple associative algebras, Alg. Logic 15 (1976), $117-142$. 
5. L.A. Bokut, Gröbner-Shirshov basis for the Braid group in the Artin-Garside generators, J. Symb. Comp. 43 (2008), 397-405.

6. Gröbner-Shirshov basis for the Braid group in the Birman-Ko-Lee generators, J. Alg. 321 (2009), 361-376.

7. L.A. Bokut and A. Vesnin, Gröbner-Shirshov bases for some Braid groups, J. Symb. Comp. 41 (2006), 357-371.

8. B. Buchberger, An algorithm for finding a basis for the residue class ring of a zero-dimensional ideal, Ph.D. thesis, University of Innsbruck, Innsbruck, Austria, 1965.

9. Y. Chen and C. Zhong, Gröbner-Shirshov bases for HNN extentions of groups and for the alternating group, Comm. Alg. 36 (2008), 94-103.

10. E.G. Karpuz, Gröbner-Shirshov bases of some semigroup constructions, Alg. Colloq. 22 (2015), 35-46.

11. C. Kocapinar, E.G. Karpuz, F. Ateş and A.S. Çevik, Gröbner-Shirshov bases of the generalized Bruck-Reilly *-extension, Alg. Colloq. 19 (2012), 813-820.

12. K. Saito, Extended affine root systems I, Coxeter transformations, Publ. Res. Inst. Math. Sci. 21 (1985), 75-179.

13. K. Saito and T. Takebayashi, Extended affine root systmes III, Elliptic Weyl groups, Publ. Res. Inst. Math. Sci. 33 (1997), 301-329.

14. A.I. Shirshov, Some algorithmic problems for Lie algebras, Siber. Math. J. 3 (1962), 292-296.

15. T. Takebayashi, Defining relations of the Weyl groups for extended affine root systems $A_{l}^{(1,1)}, B_{l}^{(1,1)}, C_{l}^{(1,1)}, D_{l}^{(1,1)}$, J. Alg. 168 (1994), 810-827.

16. Relations of the Weyl groups of extended affine root systems $A_{l}^{(1,1)}$, $B_{l}^{(1,1)}, C_{l}^{(1,1)}, D_{l}^{(1,1)}$, Proc. Japan Acad. 71 (1995), 123-124.

17. Proc. Japan Acad. 81 (2005), 51-56.

18. Poincare series of the Weyl group of the elliptic root systems $A_{l}^{(1,1)}$, $A_{l}^{(1,1) *}$ and $A_{2}^{(1,1)}$, J. Alg. Comb. 17 (2003), 211-223.

Karamanoglu Mehmetbey University, Kamil Özdag Science Faculty, Department of Mathematics, 70100, Karaman, Turkey

Email address: eylem.guzel@kmu.edu.tr

Balikesir University, Faculty of Art and Science, Department of Mathematics, 10100, Balikesir, Turkey

Email address: firat@balikesir.edu.tr

Selçuk University, Faculty of Science, Department of Mathematics, 42075, Konya, Turkey

Email address: sinan.cevik@selcuk.edu.tr 